\title{
Chapter 9 \\ Do Connections Make Systems Robust? A New Scenario for the Complexity-Stability Relation
}

\author{
Takashi Shimada, Yohsuke Murase, and Nobuyasu Ito
}

\begin{abstract}
Whether interactions among the elements make the system robust or fragile has been a central issue in broad range of field. Here we introduce a novel type of mechanism which governs the robustness of open and dynamical systems such as social and economical systems, based on a very simple mathematical model. This mechanism suggest a moderate number $(\sim 10)$ of interactions per element is optimal to make the system against successive and unpredictable disturbances. The relation between this very simple model and more detailed nonlinear dynamical models is discussed, to emphasize the relevance of this newly reported mechanism to the real phenomena.
\end{abstract}

\subsection{Introduction}

Most real complex systems of our interest are ecosystem-like. Good examples are reaction networks and gene regulatory networks in living organisms in evolutionary time scale, brain and immune system in developmental timescale, engineering systems with decentralized control scheme, ecosystems of companies or products, and

\footnotetext{
T. Shimada $(\bowtie)$

Hongo, Bunkyo-ku, Tokyo 113-8656, Japan

JST CREST, 4-1-8 Honcho, Kawaguchi, Saitama 332-0012, Japan

e-mail: shimada@ap.t.u-tokyo.ac.jp

Y. Murase

JST CREST, 4-1-8 Honcho, Kawaguchi, Saitama 332-0012, Japan
}

Department of Applied Physics, Graduate School of Engineering, The University of Tokyo, 7-3-1

RIKEN AICS, 7-1-26, Minatojima-minami-machi, Chuo-ku, Kobe, Hyogo 650-0047, Japan e-mail: yohsuke.murase@ riken.jp

N. Ito

Department of Applied Physics, Graduate School of Engineering, The University of Tokyo, 7-3-1 Hongo, Bunkyo-ku, Tokyo 113-8656, Japan

JST CREST, 4-1-8 Honcho, Kawaguchi, Saitama 332-0012, Japan

RIKEN AICS, Minatojima-minami-machi, Chuo-ku, Kobe, Hyogo 650-0047, Japan e-mail: ito@ap.t.u-tokyo.ac.jp 
social communities. In those ecosystem-like systems, there is no top-down or centralized mechanism for the system's growth and maintenance. And their complexity emerges as a result of successive introductions of new elements. In the following, we focus on the universal aspects of robustness of such ecosystem-like systems.

The robustness (or stability, fragility, resilience, etc.) of complex systems itself is indeed a classical problem [1]. Essential theoretical findings those have been found on this issue include the general instability of large and densely interacting systems [2], the self-organized criticality [3], and the relation between the robustness and the network structure of the systems $[4,5]$. However, the key and universal feature of the real complex systems, openness, has not been well considered. Meanwhile, theoretical studies on ecosystems using various different models have indicated that they share universal behaviors independent of the detail of the dynamics [6-10]. Therefore it is natural to ask how can such ecosystem-like system grow to more complex structure by adding new elements to it, using a simpler model. In the following, we first introduce a minimal model for this problem and show that it yields a novel type of transitions, together with its underlying mechanism [11]. Then we show an example of direct relation between the minimal model and the more detailed nonlinear dynamical models, which corroborates the relevance of the newly found mechanism to the real phenomena.

\subsection{A Universal Relation Between Robustness and Connection}

\subsubsection{A Minimal Model of Evolving Open Systems}

We here introduce a minimal model of evolving open systems [11]. In this model, the entire system is structured as a collection of nodes connected by directed and weighted links (Fig. 9.1). The nodes may represent various kinds of species (e.g. chemical species, different genes and proteins, neurons, animal species, companies, products, individuals, etc.). In the following, we simply call them species. Also the links may represent diverse kinds of interactions (or inputs, signal, influences, effects, etc.) among them. The directed link from species $j$ to species $i$ with its link weight denotes the influence of species $j$ on species $i$. Each species has only one property, fitness, which is simply determined by the sum of its incoming interaction weights from other species in the system. Only the rule intrinsic to the system is that each species can survive as long as its fitness is greater than zero, and otherwise it goes extinct. If the minimum fitness in the system is non-positive, we delete that species (therefore totally isolated species cannot survive). Because this extinction will modify the fitness of the other species, we re-calculate the fitness and re-identify the least-fit species. We continue this deletion procedure until the minimum fitness becomes positive, meaning that the system is stable. Once the system gets to a stable state, nothing will happen in terms of this intrinsic fast process. Therefore 


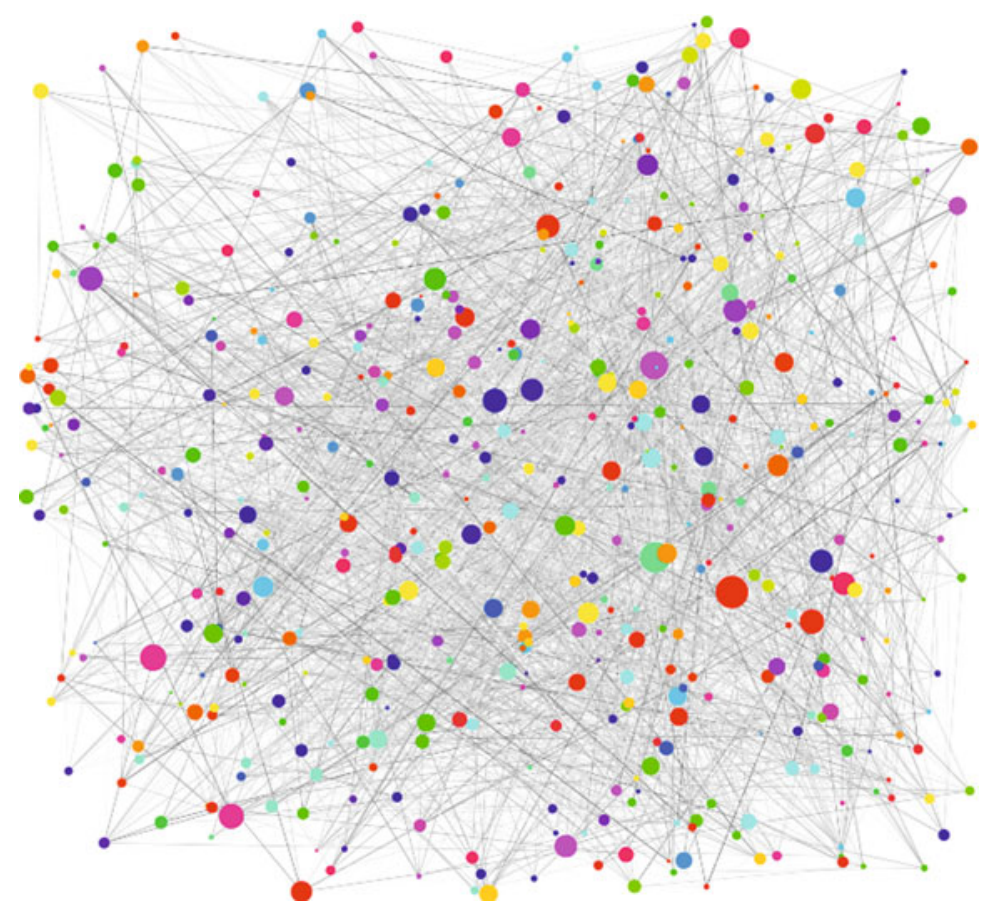

Fig. 9.1 A snapshot of ecosystem-like system obtained from the minimal model described in the Sect. 9.2.1. Nodes and links represent the general gspeciesh and ginteractionsh respectively. While the diameter of each cnode depicts the current fitness, its color is just for visibility

we proceed the time by the order of magnitude of longer unit i.e. the evolutionary time scale (in some other systems, it corresponds to the developmental time scale and so on). At each evolutionary time step $t$, a new species is added into the system. We establish interactions from and to the newly added species. The interacting species are chosen randomly from the resident species with equal probability, and the directions of the interactions are also determined randomly. The link weights are assigned randomly from a zero-mean distribution (for example, the standard normal distribution). Then, we re-calculate the fitness of each species to find whether the system can accommodate the new species or some species should become extinct. We repeat this addition-and-deletion steps. Note that the behavior of the system after a sufficiently large number of time steps does not depend on the initial condition. Therefore, this model has only one relevant parameter: $m$, the number of interactions per species. For clarity, we show below the pseudo-code of this model.

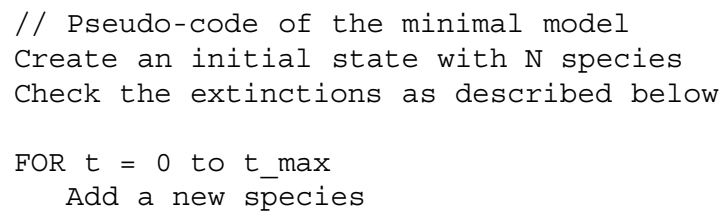




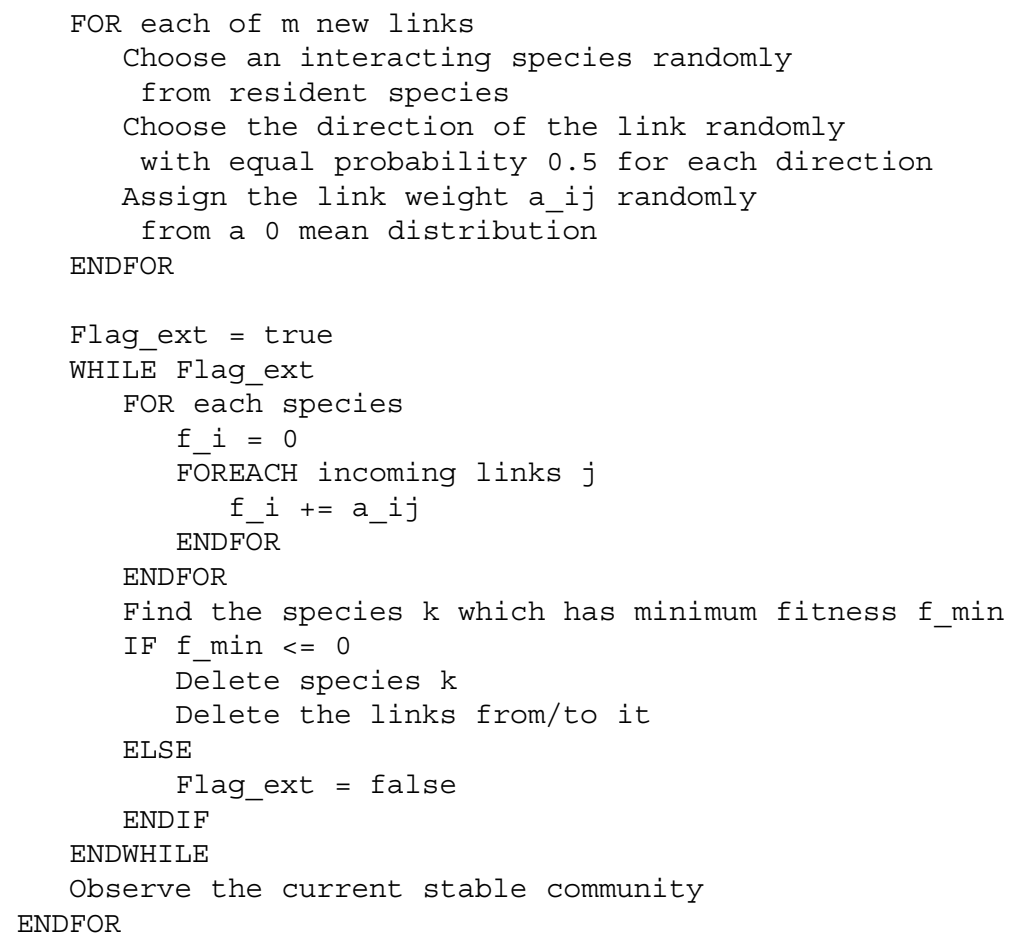

\subsubsection{Transition in Growth Behavior}

In the present model, the essential features of the ecosystem-like systems, the introduction of a new species and the interaction-dependent survival condition for each species, are took into account. And because the both processes are introduced in neutral way, i.e. giving no apparent advantage to grow or collapse. Therefore whether the system can grow under such process will purely illuminate the relation between the system's complexity and robustness. Simulation results indeed give a fascinating answer: both of the growth and collapse can happen, depending on the only one model parameter $m$. The system can grow to infinitely large size if the number of interactions per species is in a moderate range (for the case of taking the standard distribution for link weights, the range is $5 \leq m \leq 18$ ), and, if not, it stays in a finite size (Fig. 9.2). 


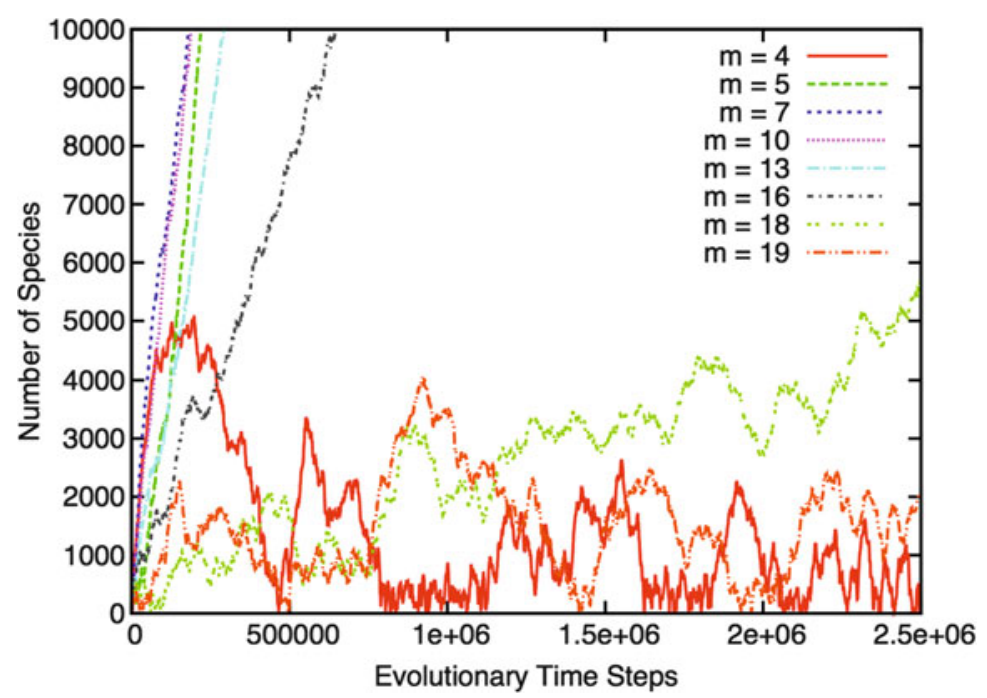

Fig. 9.2 Temporal evolutions of the number of species. The number of species diverges if $5 \leq$ $m \leq 18$. For more precise and reliable determination of the transition point in this behavior needs systematic and longer simulations (see [11])

\subsubsection{A Mean-Field Analysis and the Transition Mechanism}

The first transition at between $m=4$ and 5 turns out to be related to a kind of percolation threshold: the emergent system with too sparse interactions can have only tree-and-cycle-like network, and therefore it is too fragile to continue growing. But then why we have another transition in the denser interaction regime? This latter transition is non-trivial and novel, and therefore we mainly focus on this in this paper.

To consider the mechanism of the transition, we first investigate the topology of the emerging network. We can confirm that there is no strong structure in the emerging networks (Fig. 9.1). In other words, the structure of the emerging system remains almost random network with average degree $\sim m$. From this observation, a theoretical analysis based on a mean-field picture has been performed $[11,12]$. In this theory, we only treat the distribution function of the fitness of the species in the entire community. Because the fitness distribution function (FDF) is dependent on the parameter $m$, we write the FDF of fitness $x$ as $F(m, x)$. FDF of the newly introduced species, which has $m / 2$ incoming links on average, is easily calculated as the positive half of the normal distribution with variance $\frac{m}{2}$ :

$$
F_{0}(m, x)=\left\{\begin{array}{ll}
0 & (x \leq 0) \\
2 G\left(\sigma_{m}, x\right) & \text { ensuremath }(x \geq 0)
\end{array}, \quad \sigma_{m}=\sqrt{\frac{m}{2}} .\right.
$$


Where $G(\sigma, x)$ denotes the normal distribution with its deviation $\sigma$. After settling to the system, the species will experience either obtaining a new link from a newly introduced species or loosing a link during the extinction of interacting species. Those processes change the fitness of the species, and hence the distribution function. This change in FDF is found to be the one step of random walk with negative drift whose strength is proportional to $1 / \mathrm{m}$. Therefore, writing this process by an operator $\hat{\mathscr{D}}$, the (not normalized) FDF of species those have been experienced a loss or addition of one incoming link can be calculated from $F_{0}$ as,

$$
F_{1}(m, x)=\hat{\mathscr{E}} \hat{\mathscr{D}} F_{0}(m, x)
$$

where $\hat{\mathscr{E}}$ is the extinction operator which cut the negative part of any function:

$$
\hat{\mathscr{E}} h(x)= \begin{cases}0 & (x \leq 0) \\ h(x) & (x \geq 0)\end{cases}
$$

Note that the operators $\hat{\mathscr{D}}$ and $\hat{\mathscr{E}}$ are non-commutative. In the following we call the suffix $g$ of $F_{g}$, the number of incoming link addition/deletion events that species has experienced, as generation. As we have seen, calculation of the FDF of generation $g$ needs the FDF of younger generation, $g-1$ :

$$
F_{g}(m, x)=\hat{\mathscr{E}} \hat{\mathscr{D}} F_{g-1}(m, x)
$$

Only after performing the iterative calculation, we obtain the probability distribution function of the fitness of the entire system,

$$
F(m, x)=\frac{\sum_{g=0}^{\infty} F_{g}(m, x)}{\sum_{g=0}^{\infty} n_{g}(m)} \quad\left(n_{g}(m)=\int_{0}^{\infty} F_{g}(m, x) d x\right)
$$

which contains all the information we need under the mean-field approximation. The most important outcome from the FDF is the average probability of entire resident species going extinct during one link addition/deletion event $E$, which is calculated as

$$
E(m)=1-\int_{0}^{\infty} \hat{\mathscr{E}} \hat{\mathscr{D}} F(m, x) d x=\frac{\int_{0}^{\infty} F_{0}(m, x) d x}{\sum_{g=0}^{\infty} n_{g}(m)}=\frac{1}{\sum_{g=0}^{\infty} n_{g}(m)}
$$


From this calculation, we find that $E$ is a decreasing function of $m$. Therefore the robustness of each species against the disturbance increases with $m$.

What should be emphasized, however, is that the robustness of each species does not directly determine the robustness of the entire system. Let us see this using an infinitely large graph in which all the nodes have $m$ links. The average number of species that go extinct directly because of an inclusion of the new species is simply calculated as $m E / 2$. Because those extinctions may also trigger sequential extinctions, the expectation value of the total number of extinctions per inclusion of one species $N_{E}$ is simply calculated from an infinite geometric series as

$$
N_{E}=\sum_{n=1}^{\infty}\left(\frac{m E}{2}\right)^{n}=\frac{m E}{2-m E}
$$

Therefore the robustness of the entire system is a function of $m E$, not the bare $E$. And because $N_{E}=1$ means that the average number of extinctions balances with the number of inclusions in the long time average, that corresponds to the transition point of the growth behavior. In other words, the following self-consistent condition should be satisfied for the critical number of interactions per species: $m_{*} E\left(m_{*}\right)=1$.

Let us now focus on the relevant parameter in the argument above, $m E$. We find that the decrease of $E$ is slower than $1 / m$ (roughly $\sim 1 / \sqrt{m}$ ). Therefore $m E$ is a sub-linearly increasing function of $m$, and it crosses the critical value 1 around $m_{*}=13$. This means that the mean-filed treatment can explain the transition in the growth behavior of the system. In addition, this theory give us the simple understanding of the transition mechanism. It originates from the balance of the two effects: although having more interactions makes each species robust against the disturbances (addition and extinction of the species relating to that species), it also increases the impact of the loss of a species. In consistent with this success in explaining the transition by the mean-field analysis, we can find essentially same phase diagram in slightly modified models, such as the model with giving a randomly distributed degrees for the newly added species, the one with different distribution functions for the link weights, and so on [11].

In the classical diversity-stability relation based on the linear stability of dynamical systems, an intrinsic stability is assumed for each element to ensure the stability of each element when that has no interactions. For the system to remain stable, each element may have essentially only one interaction that is not weak comparing to the given intrinsic stability [2]. In the present mechanism, we do not assume any kind of intrinsic stability to the elements: an element with no interaction immediately goes extinct. Even so, the system with 10 or more interactions per element can grow. In this sense, the condition we have identified is very realistic. Indeed in the real systems, it is quite often to find moderately sparse networks: the average degree is in the order of 10 , not order of 1 , and that seems not dependent on the system size. This novel relation between the connection in the system and its robustness might be a origin of this. 


\subsection{The Relation with More Complex Dynamical Models}

We have reviewed a novel relation between the system's robustness and the connections in it using a very simple model. In our simple model, the extinction condition $f_{i} \leq 0$ represents the system's intrinsic dynamics. The simplicity of the model is good in terms of universality, and hence especially good for applying to social and economic systems because it is very hard to obtain precise equation of motion or evolution rule of those. And the fact that we can find a good agreement between the model and real systems in their statistics encourages us to put more emphasis on universality. A good example is lifetime distribution function of the species $[13,14]$.

However, for each certain problem, we generally treat more complex models. Therefore it would be nice if we can argue more directly about the connection between our simple model and complex models. In the following, we will consider a certain class of population dynamics models and show that the necessary condition to have extinctions in it reduces to the extinction rule in the simple model.

\subsubsection{The Extinction Condition in Population Dynamics Models}

In many dynamical models, each element has more properties in addition to its mere existence and the interactions depend on those properties. One of the most popular class is population dynamics models, in which each element has its property, population $x_{i}$. The general form of the dynamical equation of motion of population dynamics models can be written as,

$$
\dot{x}_{i}=f_{i}\left(x_{1}, x_{2}, \cdots, x_{N}\right),
$$

where the dynamical variable $\left\{x_{i}\right\}$ denote the population of element $i$. To know which species will go extinct is generally a difficult problem, because one needs to have the trajectory. This is one of the reason why so many studies substitute the stability of the system for its linear stability, which is, strictly speaking, neither enough condition nor necessary condition to really determine the fate of the species. The necessary condition to have an extinction of certain species is relatively easier, because it is at least describable simply: the necessary condition to go extinct is to satisfy

$$
\lim _{x_{i} \rightarrow 0} \dot{x}_{i}=f_{i}\left(x_{1}, \cdots, x_{i-1}, 0, x_{i+1}, \cdots, x_{N}\right)<0
$$

at somewhere in the $x_{i}=0$ surface. Such condition is again generally difficult to access and also different from the linear stability condition. 


\subsubsection{Ratio-Dependent Interactions}

The interaction term in the population dynamics with the form of $f_{i j}\left(\frac{x_{i}}{x_{j}}\right) x_{j}$, in which the predation rate per predator $j, f_{i j}(\xi)$, is an arbitrary function of the ratio of the prey to the predator $x_{i} / x_{j}$, is called ratio-dependent form in theoretical ecology and regarded as a realistic model of the predation interaction [15]. A typical simple example of the form of $f(\xi)$ is

$$
f(\xi)=\frac{B \xi}{A+\xi},
$$

where $A$ and $B$ are constants. If we neglect many-body effects such as the competition among the predators those attack the same prey, the predator's choice on multiple preys, and so on for simplicity (otherwise the dynamical equations may become implicit), the population dynamics of such systems can be written as

$$
\dot{x}_{i}=\sum_{j} f_{i j}\left(\frac{x_{i}}{x_{j}}\right) x_{j}+\sum_{k} f_{k i}\left(\frac{x_{k}}{x_{i}}\right) x_{i}
$$

where the summations run for the predators and the preys of species $i$, respectively.

\subsubsection{The Necessary Condition to Have an Extinction Under "natural” Ratio-Dependent Interactions}

Let us next limit the case by postulating the following relatively natural features to the ratio-dependent predation rate. That is, $f(\xi)$ must go to 0 as the population of the prey goes to 0 and that must saturate at a certain value when the population of the prey is abundant, i.e.

$$
\lim _{\xi \rightarrow 0} f_{i j}(\xi)=0 \cap \lim _{\xi \rightarrow \infty} f_{i j}(\xi)=b_{i j}
$$

where $b_{i j}$ represents the maximum predation rate on that interaction. The example we have seen in Eq. (9.10) satisfies these both features. And if we suppose it does not have any singularity around 0 , we can obtain its Maclaurin series as,

$$
f_{i j}\left(\frac{x_{i}}{x_{j}}\right) x_{j}=\left[\sum_{n=1}^{\infty} c_{n}^{i j}\left(\frac{x_{i}}{x_{j}}\right)^{n}\right] x_{j} .
$$


Where

$$
c_{n}^{i j}=\left.\frac{1}{n !} \cdot \frac{d^{n} f_{i j}(\xi)}{d \xi^{n}}\right|_{\xi \rightarrow 0}
$$

is the coefficient of Taylor series expansion at 0 . This means that the necessary condition for the extinction [Eq. (9.9)] of species $i$ in this model is indeed not dependent on the populations of the surrounding species:

$$
\begin{aligned}
\lim _{x_{i} \rightarrow 0} \dot{x}_{i} & =\lim _{x_{i} \rightarrow 0}\left[\sum_{j}\left\{\sum_{n=1}^{\infty} c_{n}^{i j}\left(\frac{x_{i}}{x_{j}}\right)^{n} x_{j}\right\}+\sum_{k} f_{k i}\left(\frac{x_{k}}{x_{i}}\right) x_{i}\right] \\
& =\left[\sum_{j} c_{1}^{i j}+\sum_{k} b_{k i}\right] x_{i}<0 .
\end{aligned}
$$

And this condition, $\sum_{j} c_{1}^{i j}+\sum_{k} b_{k i}<0$, that says summation of the populationindependent coefficients assigned to the interacting links should be negative, is exactly in the same class with the minimal model we introduced in Sect. 9.2.1.

\subsection{Conclusion}

We have reviewed the simple and universal mechanism of determining the robustness, and therefore its ability to grow, of ecosystem-like systems by introducing a simple model. It has been also shown that the necessary condition for extinctions in a certain type of dynamical models essentially result in the same condition with that of the simple model. This supports our future approach to verify the relevance of the newly found mechanism to the real phenomena.

Acknowledgements This work was partially supported by JSPS Grant-in-Aid for Scientific Research (C) Grant Number 15K05202.

Open Access This book is distributed under the terms of the Creative Commons Attribution Noncommercial License which permits any noncommercial use, distribution, and reproduction in any medium, provided the original author(s) and source are credited. 


\section{References}

1. MacAuthor R (1955) Fluctuations of animal populations, and a measure of community stability. Ecology 36:533-535

2. Gardner MR, Ashby WR (1970) Connectance of large dynamic (cybernetic) systems: critical values for stability. Nature 228:784

3. Bak P, Sneppen K (1993) Punctuated equilibrium and criticality in a simple model of evolution. Phys Rev Lett 71:4083-4086

4. Albert RJ, Jeong H, Barabási A-L (2000) Error and attack tolerance of complex networks. Nature 406:378-382

5. Herrmann HJ, Schneider CM, Moreira AA, Andrade JS, Havlin S (2011) Onion-like network topology enhances robustness against malicious attacks. J Stat Mech 2011:P01027

6. Taylor PJ (1988) Consistent scaling and parameter choice for linear and generalized lotkavolterra models used in community ecology. J Theor Biol 135:543-568

7. Caldarelli G, Higgs PG, McKane AJ (1988) Modelling coevolution in multispecies communities. J Theor Biol 193:345-358

8. Shimada T, Yukawa S, Ito N (2002) Self-organization in an ecosystem. Artif Life Robotics 6:78-81

9. Perotti JI, Billoni OV, Tamarit FA, Chialvo DR, Canna SA (2009) Emergent self-organized complex network topology out of stability constraints. Phys Rev Lett 103:108701

10. Murase Y, Shimada T, Ito N, Rikvold PA (2010) Random walk in genome space: a key ingredient of intermittent dynamics of community assembly J Theor Biol 264:663-672

11. Shimada T (2014) A universal transition in the robustness of evolving open systems. Sci Rep 4:4082

12. Shimada T (2015) In: Mathematical approaches to biological systems. Ohira T, Uzawa $T$ (eds) Springer, Japan, pp 95-117

13. Shimada T, Yukawa S, Ito N (2003) Life-span of families in fossil data forms q-exponential distribution Int J Mod Phys C 14:1267-1271

14. Murase Y, Shimada T, Ito N (2010) A simple model for skewed species-lifetime distributions New J Phys 12:063021

15. Drossel B, Higgs PG, Mckane AJ (2001) The influence of predator prey population dynamics on the long-term evolution of food web structure. J Theor Biol 208:91-107 